\title{
Asthma control, self-management and healthcare access during the COVID-19 Epidemic in Beijing
}

\author{
Chun Chang ${ }^{1}$, Linlin Zhang ${ }^{1}$, Fawu Dong ${ }^{1}$, Ying Liang ${ }^{1}$, Yahong Chen ${ }^{1}$, Mairipaiti \\ Abulikemu ${ }^{1}$, Ying Shang ${ }^{1}$, and Yongchang Sun ${ }^{1}$ \\ ${ }^{1}$ Peking University Third Hospital
}

June 8, 2020

Asthma control, self-management and healthcare access during the COVID-19 Epidemic in Beijing

Chun Chang ${ }^{a *}$ M.D., Linlin Zhanga*B.S., Fawu Dong ${ }^{a *}$ B.S., Ying Liang ${ }^{a}$ M.D., Yahong Chen ${ }^{a}$ M.D., Ying Shang a B.S., Mairipaiti Abulikemua B.S., Yongchang Sun ${ }^{\mathrm{a}}$ \# M.D.

a Department of Respiratory and Critical Care Medicine, Peking University Third Hospital, Beijing, China. \#, Corresponding to Yongchang Sun:

Department of Respiratory and Critical Care Medicine, Peking University Third Hospital, Beijing, China.

North Garden Rd. 49.

Haidian District, Beijing, 100191, China

Tel: +8601013910979132

Fax +861082266989

E-mail: suny@bjmu.edu.cn

* Chun Chang, Linlin Zhang and Fawu Dong contributed equally to this work;

To the Editor:

The pandemic of COVID-19, caused by the pathogen SARS-CoV-2, has now spread around the globe. Social distancing and restriction measures during COVID-19 pandemic may have impacts on asthma control and management in terms of medication availability and healthcare access. International societies responded quickly by releasing guidance on the management of asthma during the COVID-19 pandemic ${ }^{1-4}$. However, these temporary guidelines were based largely on previous asthma guidelines and expert consensus, because evidence from related studies was lacking. Therefore, we investigated the status of asthma control, selfmanagement, medications and healthcare utilization of asthma patients during the COVID-19 epidemic in Beijing, aiming to provide data for guideline recommendations on asthma managements during the emergency.

Patients with asthma, selected randomly from our hospital database, were interviewed by phone call. Detailed description of items in the questionnaire is available in this article's online supplementary material.

We contacted 286 patients, of whom 178 (62.2\%) responded with valid results. Sociodemographic data and clinical characteristics of the patients before the COVID-19 pandemic are provided as online supplements. During the COVID-19 epidemic in Beijing (January 25, 2020 to April 25, 2020), the majority (74.2\%, $132 / 178$ ) of the patients felt that their symptoms had not changed as compared with usual times, while 
$18.0 \%$ (32/178) felt better, and 7.9\% (14/178) felt worse. The mean ACT score of the 178 patients was 22.76 \pm 3.06 (ranging from 8 to 25) in the last 4 weeks before the survey. According to the criteria of ACT scoring from GINA, asthma was classified as well-controlled in $89.3 \%$, not well-controlled in $6.2 \%$, and very poorly controlled in $4.5 \%$ of the patients. During this period, only $24.7 \%(44 / 178)$ of the patients had ever visited a hospital or clinic for asthma, of whom 11 patients had 2 visits, and 6 had [?]3 visits, totaling 74 visits. $14.9 \%(11 / 74)$ of all medical visits were due to exacerbation of asthma, while the remaining visits $(63 / 74$, $85.1 \%$ ) were for regular prescription of asthma medications. Only 6 patients (3.4\%) sought consultation online. (Table 1)

Notably, 25.6\% (45/176) of the patients experienced aggravation of asthma symptoms during the COVID-19 epidemic, but $75.6 \%(34 / 45)$ of them did not see a doctor, because $67.6 \%(23 / 34)$ of the patients thought that they did not need to go to the hospital and took more medications by themselves, and the remaining $32.4 \%$ (11/34) worried about cross-infection of COVID-19 in the hospital. No patient said that they did not see a doctor because they could not arrange an appointment. Eleven patients went to the hospital due to aggravation, $81.8 \%(9 / 11)$ to the outpatient, while only $18.2 \%(2 / 11)$ to the Emergency Department (ED).

Table 1 Asthma control and management during the COVID-19 epidemic in Beijing

\begin{tabular}{|c|c|c|c|}
\hline & $N$ & $n(\%)$ & $95 \%$ CI $(\%)$ \\
\hline Perception of overall control of asthma & 178 & & \\
\hline No change & & $132(74.2 \%)$ & $67.7-80.7$ \\
\hline Improve & & $32(18.0 \%)$ & 12.3-23.7 \\
\hline Aggravation & & $14(7.9)$ & $3.9-11.9$ \\
\hline ACT score & 178 & & \\
\hline $20-25$ & & $159(89.3 \%)$ & 84.7-93.9 \\
\hline $16-19$ & & $11(6.2 \%)$ & $2.6-9.8$ \\
\hline $5-15$ & & $8(4.5 \%)$ & $1.4-7.6$ \\
\hline Number of medical visits due to asthma & 178 & & \\
\hline 0 & & $134(75.3 \%)$ & $68.9-81.7$ \\
\hline 1 & & $27(15.2 \%)$ & $9.8-20.5$ \\
\hline 2 & & $11(6.2 \%)$ & $2.6-9.8 ? \gtreqless ?$ \\
\hline 3 & & $6(3.4 \%)$ & $0.7-6.0$ \\
\hline
\end{tabular}

Scores of 20-25 are classified as well-controlled asthma; 16-19 as not well-controlled; and 5-15 as very poorly controlled asthma. $N$, total number of qualified subjects for evaluation; $n(\%)$, frequency (percentage of frequency); $95 \%$ CI, 95\% confidence interval.

Of note, during the COVID-19 epidemic, 13.5\% (24/178) of the patients had worried about insufficient maintenance medications, among whom $45.8 \%(11 / 24)$ had reduced medication dosing for this reason. After reducing medication dosing, $27.3 \%(3 / 11)$ of them experienced asthma aggravation. Among patients who had their prescriptions refilled during the COVID-19 epidemic, $60.6 \%(40 / 66)$ chose to go to a hospital, $36.4 \%(24 / 66)$ chose a nearby pharmacy, and $3.0 \%(2 / 66)$ chose to purchase online.

For patients on maintenance therapy $(\mathrm{n}=105), 93.3 \%$ (98/105) used two or even three drugs, as illustrated in Table 2. ICS plus LABA was the most commonly used therapy, followed by ICS/LABA plus oral leukotriene modifiers.

Table 2 Current maintenance therapy during the COVID-19 pandemic

\begin{tabular}{llll}
\hline & $N$ & $n(\%)$ & $95 \%$ CI (\%) \\
\hline Maintenance medications & 105 & & \\
ICS/LABA & & $96(91.4 \%)$ & $86.0-96.9$
\end{tabular}




\begin{tabular}{|c|c|c|c|}
\hline & $N$ & $n(\%)$ & $95 \%$ CI $(\%)$ \\
\hline ICS & & $3(2.9 \%)$ & $0.0-6.1$ \\
\hline Oral theophylline & & $4(3.8 \%)$ & $0.1-7.5$ \\
\hline Inhaled Short-acting $\beta$-agonist (SABA) & & $7(6.7 \%)$ & $1.8-11.5$ \\
\hline Oral leukotriene modifiers & & $25(23.6 \%)$ & $15.5-32.1$ \\
\hline Inhaled anticholinergics & & $2(1.5 \%)$ & $0.0-4.6$ \\
\hline Oral antihistamines & & $4(3.8 \%)$ & $0.1-7.5$ \\
\hline
\end{tabular}

$N$, total number of qualified subjects for evaluation; $n(\%)$, frequency (percentage of frequency); $95 \% \mathrm{CI}$, $95 \%$ confidence interval. $M \pm \mathrm{SD}$, average \pm standard deviation.

Our survey found that, during the COVID-19 epidemic in Beijing, the majority of our asthma patients (89.3\%) had their disease controlled as defined by GINA, which is higher than the results of general population before the pandemic. A multi-center, retrospective, cross-sectional study in China ${ }^{5}$ indicated that less than one-third $(28.7 \%)$ of the patients had controlled asthma, and the control rate in Beijing was $31.4 \%$. We speculate that this might be due to social distancing and mandatory closure of places where aggregation may occur, stepped-up public hygiene measures and the wearing of masks during the COVID-19 epidemic, thereby reducing contact with allergens and viruses.

During the study period, a quarter of our patients experienced an exacerbation of asthma, which is more than the general population before the COVID-19 pandemic. A cross-section study in China showed that the proportion of people with asthma experiencing an exacerbation in the previous year before the study was $15.5 \%$ (4). The patients enrolled in our study had a higher rate of asthma exacerbation but lower rates of ED visit and hospitalization. It is not surprising to see that one-third of them worried about the risk of exposure to SARS-CoV-2 in the hospital, although most patients regarded their symptoms as not severe and could be relieved by self-management with medications.

In our survey, the majority of the patients used ICS plus LABA as maintenance therapy, which is consistent with guideline recommendations ${ }^{1,2,4}$. Indeed, there is no evidence regarding whether currently available asthma and allergy treatments, including corticosteroids and bronchodilators, increase the susceptibility to or severity of COVID-191. On the contrary, it may be more likely that a patient with asthma would have an exacerbation from other causes, including seasonal pollen exposure or a virus other than SARS-CoV-2 if they stopped regular use of indicated controller therapy. An exacerbation may drive asthmatic patients to seek medical treatment, which would put them at increased risk of being exposed to SARS-CoV-2 during the current pandemic ${ }^{4}$.

The use of telehealth in asthma treatment in recommended within a risk-stratified context of the SARS-CoV2 pandemic ${ }^{4}$. However, in our interview, only a few patients used online consultation during the COVID-19 pandemic. Patients with mild-to-moderate or well-controlled asthma were encouraged to use digital medicine services $^{6}$. Outpatient service should be prioritized for patients who have poorly controlled asthma, have worsening asthma symptoms, or who have required dose escalations of their asthma medications in the past several months' time ${ }^{4}$.

In conclusion, our survey revealed the status of asthma control, exacerbations, self-management and healthcare utilization during the COVID-19 epidemic in Beijing, which supports the recommendation that patients continue taking their prescribed asthma medications as usual and maintain good asthma control during the ongoing pandemic. For containment of viral transmission, social distancing is being encouraged, but measures should be taken to mitigate the negative impact on asthma.

Chun Chang a M.D.

Linlin Zhang ${ }^{a}$ B.S.

Fawu Dong a B.S. 
Ying Liang a M.D.

Yahong Chen ${ }^{a}$ M.D.

Ying Shang ${ }^{\text {a }}$ B.S.

Mairipaiti Abulikemu ${ }^{a}$ B.S.

Yongchang Sun ${ }^{a}$ M.D.

From ${ }^{a}$ Department of Respiratory and Critical Care Medicine, Peking University Third Hospital, Beijing, China.

Correspondence

Department of Respiratory and Critical Care Medicine, Peking University Third Hospital, Beijing, China.

North Garden Rd. 49.

Haidian District, Beijing, 100191, China

E-mail: suny@bjmu.edu.cn

\section{Acknowledgments}

This study was supported by the National Natural Science Foundation of China. [No. 81970028]

\section{Conflict of interest}

The authors declare that they have no relevant conflicts of interest.

\section{References}

1. Global Initiative for Asthma. Global Strategy for Asthma Management and Prevention, 2020. Available from:https://ginasthma.org/. Accessed May 9, 2020.

2. National Institute for Health and Care Excellence. COVID-19 rapid guideline: severe asthma. Available from:https://www.nice.org.uk/guidance/ng166. Accessed May 9, 2020.

3. National Health Service. Clinical guide for the management of respiratory patients during the coronavirus pandemic. Available from:https://www.england.nhs.uk/coronavirus/publication/specialtyguides/. Accessed May 9, 2020.

4. Shaker MS, Oppenheimer J, Grayson M, Stukus D, Hartog N, Hsieh E, et al. COVID-19: Pandemic Contingency Planning for the Allergy and Immunology Clinic. J Allergy Clin Immunol Pract . 2020;8(5):1477-1488.e5. doi:10.1016/j.jaip.2020.03.012

5. Su N, Lin J, Chen P, Li J, Wu C, Yin K, et al. Evaluation of asthma control and patient's perception of asthma: findings and analysis of a nationwide questionnaire-based survey in China. J Asthma . 2013;50(8):861-870. doi:10.3109/02770903.2013.808346

6. Centers for Disease Control and Prevention (U.S.). Coronavirus Disease 2019 (COVID-19)/People Who Need Extra Precautions/People Who Are At Higher Risk/People with Moderate to Severe Asthma. Available from:https://www.cdc.gov/coronavirus/2019-ncov/need-extra-precautions/index.html. Accessed May 9, 2020. 